\title{
Stefan Morent
}

\section{»Eruditio una cum pietate iuncta« - Zu Glareans Choralverständnis}

In Kapitel 38 des zweiten Buches seines Dodekachordon, das dem Verhältnis zwischen einstimmigem Choral und mehrstimmiger Figuralmusik gewidmet ist, spricht sich Glarean vehement für die Beibehaltung des Choralgesangs im Gottesdienst aus. Seine voller Emphase vorgetragene Apologie gipfelt in der Bemerkung, nie sei etwas Vollkommeneres als das Repertoire der Gesänge für die Messe im Graduale geschaffen worden:

At ego (libere enim pronuntiandum est quod sentio) Ecclesiasticum cantum tantum abest, ut aboleri uelim, ut asseuerem, nihil absolutius unquam natum, quippe in quo, qui composuerunt, eruditionem unà cum pietate iunctam manifestissimis argumentis ostenderint, loquor de Missarum corpore (Graduale uocant) in quo se licissime omnes tractarunt Modos, nec tractarunt solum sed pro materia rebus applicuerunt, atque ita natiuam Modorum Indolem (ut sic loquar) expresserunt, ut nihil perfectius ab homine fieri potuisse satis notum sit ijs, qui has res non suo affectu, ut nunc fit, sed arte ac adhibito iudicio estimant. ${ }^{1}$

Aber ich, denn ich muss frei aussprechen, was ich denke, bin so weit davon entfernt, mir den kirchlichen Gesang abgeschafft zu wünschen, dass ich versichere, dass nie etwas Vollkommeneres geschaffen wurde, da in demselben diejenigen, die ihn komponierten, auf deutlichste Weise Gelehrsamkeit verbunden mit Frömmigkeit bekundet haben; ich spreche vom Corpus der Messgesänge (Graduale genannt), in welchem sie mit äußerst glücklicher Hand alle Modi behandelt, und nicht nur behandelt, sondern sie als Ausgangsmaterial für die Umsetzung verschiedener Inhalte angewendet und damit die natürliche Anlage (sozusagen) der Modi zum Ausdruck gebracht haben, so dass jenen, die diese Dinge nicht nach ihrer Neigung, wie es jetzt geschieht, sondern mit Kunstverständnis und ernsthaftem Urteil abwägen, hinreichend bekannt sein muss, dass nichts Vollkommeneres von Menschen je hätte geschaffen werden können.)

Hier lassen die Formulierungen "qui composuerunt" und »ab homine fieri« in besonderem $\mathrm{Maße}$ aufhorchen, zeigen sie doch eine Akzentverschiebung im Choralverständnis gegenüber der Tradition an, die bemerkenswert ist. Zwar sollte mit dem Begriff der Modernität zumal in früheren Epochen vor-

1 Dod., S. 176. 
sichtig umgegangen werden, ${ }^{2}$ und Leo Treitler empfiehlt zu Recht, hier vermeintlich vertrauten Konzepten gegenüber zunächst misstrauisch zu sein, denn es könnte sich dahinter der Wunsch verbergen, das entdecken zu wollen, was man bereits kennt. ${ }^{3}$ Aus obigem Zitat wird aber doch deutlich, dass Glarean von der Vorstellung ausgeht, der Choral sei das Ergebnis menschlichen Tuns im Sinne eines kompositorischen Aktes, dessen Spuren sich unter anderem in der adäquaten Handhabung der Modi nachvollziehen lassen. ${ }^{4}$

Diese Sichtweise unterscheidet sich wesentlich von der älteren, seit der Karolingerzeit und bis in die Gegenwart Glareans hinein tradierten, ideologisch motivierten Vorstellung, der Choral sei ein vom Heiligen Geist inspiriertes Melodiengut, das seiner sakrosankten Natur wegen menschlichem Zugriff gerade entzogen sei. Um diesen Unterschied deutlicher zu machen, dürfte es hilfreich sein, zunächst die ältere Tradition anhand einiger weniger Stationen zu beleuchten.

Die um 900 aufkommende Legende von Papst Gregor dem Großen, dem der Choral vom Heiligen Geist in Gestalt einer Taube eingegeben wird, und die ihren Niederschlag in Dichtung, Musik und bildlicher Darstellung gefunden hat, spiegelt das ältere Choralverständnis wider. ${ }^{5}$ Gleichzeitig dient die Figur Gregors als »organum«bzw. »habitaculum spiritus sancti ${ }^{6}$ als Prototyp für andere sinspirierte Melodienschöpfer wie etwa Hildegard von Bingen oder Hermann Joseph von Steinfeld. ${ }^{7}$ Aurelian von Réôme stellt Gregor den Hl. Ignatius an die Seite, der des Gesangs der Engel im Himmel teilhaftig wurde und durch dessen Weitergabe den kirchlichen Gesang mit begründet hätte. ${ }^{8}$ Obwohl sie mit Namen genannt werden, gelten Gregor

2 Vgl. hierzu Joachim Heinzle, "Einleitung: Modernes Mittelalter «, in: Modernes Mittelalter. Neue Bilder einer populären Epoche, hrsg. von Joachim Heinzle, Frankfurt 1994, S. 9-13.

3 Leo Treitler, With Voice and Pen. Coming to know medieval song and how it was made, Oxford 2003, S. ix.

4 Zur Problematik der Konzepte von "Komposition" und "Komponist" im Mittelalter vgl. Andreas Haug, »Der Beginn europäischen Komponierens in der Karolingerzeit: Ein Phantombild", in: Die Musikforschung 58 (2005), S. 225-241, insbes. S. 225 und 239-24l.

5 Bruno Stäblein, „Gregorius Praesul, der Prolog zum römischen Antiphonale. Buchwerbung im Mittelalter«, in: Musik und Verlag. Karl Vötterle zum 65. Geburtstag, hrsg. von Richard Baum und Wolfgang Rehm, Kassel 1968, S. 554-561.

6 Ebda., S. 540 und 554.

7 Stefan Morent, "Von einer Theologie der Musik. Zur Musikanschauung bei Hildegard von Bingen «, in: Kirchenmusikalisches Jahrbuch 81 (1997), S. 31-38; Marianne Richert Pfau und Stefan Johannes Morent, Hildegard von Bingen. Der Klang des Himmels, Köln 2005 (Europäische Komponistinnen, 1), S. $275 \mathrm{f}$.

8 Vgl. Aureliani Reomensis Musica Disciplina, hrsg. von Lawrence Gushee, Rom 1975 (Corpus Scriptorum de Musica, 21), S. 129. 
und Ignatius nicht eigentlich als Autoren des Chorals, sondern vielmehr als Werkzeuge, die der Weitergabe einer Musik göttlichen Ursprungs dienen.

Ganz im Gegensatz hierzu werden einzelne der zahlreichen dichterischmusikalischen Erweiterungen des 9. und 10. Jahrhunderts in Gestalt von Tropen und Sequenzen konkreten Dichter- und Musikerpersönlichkeiten zugeordnet. Die Casus Sancti Galli berichten, ein Sänger namens Petrus habe »iubilos ad sequentias« mit der Bezeichnung Metenses, ein anderer namens Romanus solche mit der Bezeichnung Romana und Amoena verfertigt. ${ }^{9} \mathrm{Be}-$ sonders fällt hier auf, dass nicht nur einzelne Dichtermusiker als Schöpfer genannt, sondern auch konkrete Orte der Entstehung angegeben werden können: Im ersten Fall Metz, im zweiten St. Gallen. Notker Balbulus schließlich beschreibt in seinem Liber ymnorum detailliert, wie er einzelne Sequenzen geschaffen habe, sowie das dahinter stehende Kunstprinzip. ${ }^{10}$ Gegenüber dem älteren Repertoire des Chorals, das als abgeschlossen, überindividuell und der Zeit sowie menschlichen Kategorien enthoben erscheint, weisen die Neuschöpfungen offenbar Qualitäten auf, die eine Zuschreibung an einzelne Autoren, konkrete Orte und Zeiten ermöglichen. ${ }^{11}$

Diese Zweiteilung setzt sich nach der Jahrtausendwende fort, in einer Zeit, in der Neukompositionen in Gestalt von Offizien einen immer größeren Raum einnehmen und erste Ansätze zu deren theoretischer Durchdringung in Form von Kompositionslehren erscheinen. So spricht Guido von Arezzo in seinem Micrologus ganz selbstverständlich davon, dass Gregor den Tritus im Choral besonders bevorzugt hätte, ${ }^{12}$ andererseits gibt er in den Kapiteln 15 bis 17 Empfehlungen und Regeln zur rechten Fügung von Melodien. Auf welches Repertoire diese Anleitungen verweisen, bleibt unklar; jedenfalls dienen sie nicht einer nachträglichen Erklärung des älteren Choralrepertoires und bleiben, wie am Beispiel der Liqueszenz als Vortrags- und Ausdrucks-

9 Ekkehard IV. von St. Gallen, Casus Sancti Galli, Kap. 47, zit. nach Hans F. Haefele, Ekkehard IV. St. Galler Klostergeschichten, Darmstadt 1980 (Ausgewählte Quellen zur deutschen Geschichte des Mittelalters, 10), S. 108. Zur Interpretation dieser Stelle vgl. A. Haug, Der Beginn (wie Anm. 4), S. 227-229.

10 Wolfram von den Steinen, Notker der Dichter und seine geistige Welt, Editionsband: Notkeri liber ymnorum, (Bern 1948), 2. Aufl. Bern 1978, S. 8-10.

11 Vgl. Wulf Arlt, "Komponieren im Galluskloster um 900: Tuotilos Tropen >Hodie cantandus est zur Weihnacht und >Quoniam dominus Iesus Christus zum Fest des Iohannes evangelista«, in: Schweizer Jahrbuch für Musikwissenschaft Neue Folge 15 (1995), S. 41-70.

12 Vgl. Guidonis Aretini Micrologus, hrsg. von Joseph Smits van Waesberghe, Rom 1955 (Corpus Scriptorum de Musica, 4), S. $207 \mathrm{f}$. 
mittel zugleich deutlich wird, ${ }^{13}$ eng mit dem regelgerechten Vortrag im Sinne einer ursprünglichen Einheit von "cantare " und "componere " verbunden. ${ }^{14}$

Konkreter wird in dieser Hinsicht der in den ersten Jahrzehnten des 12. Jahrhunderts verfasste Traktat De musica cum tonario des Johannes (früher: J. Affligemensis / Cotto): Seine Kompositionsregeln beziehen sich zum Teil auf Neuschöpfungen im Bereich kirchlicher Gesänge, zum Teil aber offensichtlich auch auf außerliturgische Musik. ${ }^{15}$ Solche neuen Kompositionen sind im Gegensatz zum Choralrepertoire auch kritisierbar, nämlich dann, wenn sie den aufgestellten Regeln widersprechen. Die im süddeutschen Raum seit dem 11. Jahrhundert sich ausbreitende neue Auffassung der Modi mit der Verschmelzung authentischer und plagaler Formen verurteilt Johannes am Beispiel der mit Wilhelm von Hirsau verbundenen Merkmelodie "Ter terni sunt modi« als bloßen Ohrenkitzel ("pruritum aurium«) der Neutöner (»novi modulatores«). ${ }^{16}$ Das ältere Choralrepertoire für Messe und Offizium ist für ihn dagegen gemäß der Tradition fest mit den Namen Ignatius, Ambrosius und Gregor verbunden, die der Kirche den Choral mit Hilfe des Heiligen Geistes ("Spiritu Sancto ei ... assidente et dictante «) geschenkt hätten - wenn Johannes an dieser Stelle auch ein relativierendes "ut fertur" (»wie es heißt«) einfügt. ${ }^{17}$

Auch in den folgenden Jahrhunderten, in denen Neukompositionen einstimmiger Musik immer weiter zunehmen, die Mehrstimmigkeit ihren Aufstieg beginnt und neue philosophisch-theologische Strömungen auch das Nachdenken und Schreiben über Musik verändern, bleibt das tradierte Choralverständnis weitgehend unangetastet: Die wohl ins 13. Jahrhundert zu datierende anonyme Instituta patrum de modo psallendi sive cantandi $i^{18}$ spricht ebenso selbstverständlich von den heiligen Vätern, die den Choral und seine rechte Vortragsweise "per contemplationem « von den Engeln und vom Heiligen Geist empfangen hätten, ${ }^{19}$ wie Heinrich Eger von Kalkar oder ein ano-

13 Ebda., S. 174-176.

14 Karlheinz Schlager, "Ars cantandi - ars componendi. Texte zum Vortrag und zur Fügung des mittelalterlichen Choralse, in: Geschichte der Musiktheorie, Bd. 4: Die Lehre vom einstimmigen liturgischen Gesang, Darmstadt 2000, S. 219 und 239.

15 Vgl. Johannes Affligemensis, De Musica cum Tonario, hrsg. von Joseph Smits van Waesberghe, Rom 1950 (Corpus Scriptorum de Musica, 1), S. 116.

16 Vgl. ebda., S. 96.

17 Vgl. ebda., S. 115

18 Siehe K. Schlager, Ars cantandi (wie Anm. 14), S. 225.

$19 \mathrm{Vgl}$. Michael Bernhard, Clavis Gerberti. Eine Revision von Martin Gerberts Scriptores ecclesiastici de musica sacra potissimum (St. Blasien 1784), Tl. 1, München 1989 (Veröffentlichungen der Musikhistorischen Kommission der Bayerischen Akademie der Wissenschaften, 7), S. 7. 
nymer Kartäuser-Traktat am Ende des 14. Jahrhunderts. ${ }^{20}$ Die beiden Letzteren betonen gleichermaßen, dass etwaige Auffälligkeiten im älteren Choral (»regulas musicales excedere«) durch die Autorität der heiligen Väter und des seligen Gregor legitimiert seien, ${ }^{21}$ und bestätigen damit indirekt die Unantastbarkeit dieses Repertoires.

Ohne Zweifel war Glarean mit diesem mittelalterlichen Choralverständnis, wie es in den Theoretikertraktaten tradiert wurde, vertraut; hierfür sprechen schon seine zahlreichen Verweise, z. B. auf Guido von Arezzo. Und diese ungebrochene Tradition bildet auch den Hintergrund, vor dem Glareans Äußerungen gesehen werden müssen. Der Kontrast wird hierbei noch schärfer bei Texten, die zeitlich in die nähere Umgebung Glareans führen. Johannes Tinctoris verbindet bei der Diskussion der Modi in seinem Liber de natura et proprietate tonorum die Herkunft der acht »toni« mit der »disciplina Gregorii «. ${ }^{22}$ Ganz ähnlich lobt Franchino Gaffurio, den Glarean als Autorität vielfach zitiert, in seiner Practica Musica in beinahe schon schwärmerischem Ton Papst Gregor dafür, wie er Reponsorien, Antiphonen, Introitus, Alleluias, Offertorien und Communiones gemäß ihrer jeweiligen Funktion geschaffen habe, und dies alles im Einklang mit den Prinzipien der Ars musica. ${ }^{23}$ Hiermit ist ein Kontext aufgerufen, der wiederum bis zu den karolingischen Autoren zurückreicht: Die durch Gregor und die heiligen Väter übermittelten Melodien sind nicht nur göttlichen Ursprungs, in ihnen offenbart sich auch die von Ratio durchdrungene göttliche Ordnung, die in den Gesetzmäßigkeiten der Ars musica ihren Widerhall findet; ${ }^{24}$ bereits der Gregorius presul-Prolog berichtet ja, Gregor habe ein "libellum musicae artis « (»Buch, dessen Melodien den Regeln der musikalischen Kunst genügen«) zusammengestellt bzw. verfasst. ${ }^{25}$

20 Siehe K. Schlager, Ars cantandi (wie Anm. 14), S. 273 und 279.

21 Vgl. Das Cantuagium des Heinrich Eger von Kalkar (1328-1408), hrsg. von Heinrich Hüschen, Köln 1952 (Beiträge zur rheinischen Musikgeschichte, 2), S. 58f.; und Cuiusdam Carthusiensis monachi Tractatus de musica plana, zit. nach Edmond de Coussemaker, Scriptorum de musica medii aevi: nova series, Bd. 2, Paris 1867, Reprint Mailand 1931, S. 445.

22 Vgl. Johannis Tinctoris opera theoretica, Bd. 1, hrsg. von Albert Seay, Rom 1975 (Corpus Scriptorum de Musica, 22), S. 67.

23 Vgl. Practica Musica Franchini Gafori Lavdensis, Mailand 1496, Reprint Farnborough 1967, Bologna 1972 (Bibliotheca musica Bononiensis, 2.7) und New York 1979 (Monuments of Music and Music Literature in Facsimile, 2.99), Bg. bvir

24 Hierzu ausführlich: Stefan Morent, »Musicus et / versus cantor - Zum Verhältnis von Musiktheorie und Gregorianischem Choral im 9. Jahrhundert", in: Wissensstrukturen - Wissensvermittlung in der frühen Neuzeit, hrsg. von Stefan Seit und Stefan Morent, unter Mitarbeit von Andrea Geier, Stuttgart 2006 (in Vorbereitung).

25 B. Stäblein, Gregorius presul (wie Anm. 5), S. 537. 
In etwas nüchternerem Stil zitiert Conrad von Zabern in seinem Novellus musicae artis tractatus (zwischen 1460 und 1470) beinahe wörtlich den Passus aus dem Traktat des Johannes, nach dem Ignatius, Ambrosius und Gregor als die Urheber des Chorals zu gelten hätten. ${ }^{26}$ Kurz und prägnant stellt Johannes Cochläus, Glareans Lehrer an der Kölner Universität, im Kapitel seines Tetrachordum musices über den Choral fest: »Quid est Musica Plana? ... Dicitur \& Gregoriana ab inventore Gregorio Papa « (»Was ist der Choral? ... Er wird wegen seines Erfinders Papst Gregor gregorianisch genannt «). ${ }^{27}$

Fragt man nun nach den Beweggründen für Glareans Abweichen von dieser Tradition, so sind diese sicherlich zunächst in der Programmatik des Dodekachordon selbst zu suchen. Wie Glarean bereits in dessen Einleitung ausführt, versteht er das von ihm propagierte System der zwölf Modi nicht als eine Neuerung seinerseits, sondern - humanistischem Denken verpflichtet als Erhellung einer bereits in der Antike bekannten Tradition. ${ }^{28}$ Die apologetische Absicht hinter dieser Formulierung ist freilich offensichtlich: Um den potenziellen Leserkreis seiner Schrift, gelehrte Humanisten wie Kleriker gleichermaßen, zu überzeugen, war es notwendig, seine Neuinterpretation des modalen Systems mit einer Kombination aus mittelalterlicher »auctoritas« und ehrwürdiger antiker Tradition zu stützen. ${ }^{29}$ Konsequenterweise setzt er sich selbst das Ziel, seine These anhand theoretischer Erörterungen und praktischer Beispiele zugleich zu beweisen. ${ }^{30}$

26 Vgl. Conrad von Zabern, Novellus musicae artis tractatus, zit. nach Karl Werner Gümpel, Die Musiktraktate Conrads von Zabern, Wiesbaden 1956 (Abhandlungen der Geistes- und sozialwissenschaftlichen Klasse der Akademie der Wissenschaften und der Literatur, 1956.4), S. 186.

27 Zit. nach Johannes Cochlaeus, Tetrachordum musices, Nürnberg 1511, Reprint der Ausgabe Nürnberg 1512, Hildesheim 1971, Bg. B [1] $]^{\mathrm{T}}$.

28 Dod., Bg. a2 ${ }^{\mathrm{v}}$ : »Constat igitur non esse nouam rem hanc nostram de XII Modis assertionem, sed probam antiquitatis instaurationem. (»Es steht daher fest, dass unsere Darstellung der zwölf Modi nichts Neues, sondern eine wirkliche Erneuerung der Antike ist.«) Bereits in der Widmungsvorrede seiner Isagoge von 1516 benennt Glarean als sein vorrangiges Ziel, die ars musica zu ihrer antiken Hochblüte zurückführen zu wollen ("pristino splendori restituere "); Faksimile der Vorrede bei Frances Berry Turrell, "The Isagoge in musicen of Henry Glarean«, in: Journal of Music Theory 3 (1959), S. 110.

29 Vgl. Laurenz Lütteken, Art. "Glarean", in: Die Musik in Geschichte und Gegenwart, 2. Aufl., hrsg. von Ludwig Finscher, Personenteil, Bd. 7, Kassel und Stuttgart 2002, Sp. 1044.

30 Dod., Bg. a2v: "Verum id non credat Lector, donec firmis argumentis negocium ipsum per Mathematicas rationes, ac praecepta per luculentissima exempla ostendero, quod duobus posterioribus libris nos fecisse omnino persuasum habemus.« (»Aber dies wird der Leser nicht glauben, bis ich es durch mathematische Berechnungen bewiesen und die Regeln durch äußerst klare Beispiele begründet habe, was wir, wie ich völlig überzeugt bin, in den beiden folgenden Büchern geleistet haben.«) 
Nach der theoretischen Darlegung des Systems der zwölf Modi, das er durch Zitate antiker Autoren zu untermauern sucht, ist es Glarean deshalb um eine Beweisführung im Choral selbst zu tun. Wenn die zwölf Modi keine Neuerung, sondern lediglich die Wiedergewinnung einer nur verschütteten Tradition darstellen, dann müssen sie auch in den Melodien des Choralrepertoires bereits vorhanden sein, auch wenn bisher vier Modi unerkannt blieben. ${ }^{31}$ Wie wichtig Glarean diese Verankerung seines Gedankengebäudes im Kontext der christlichen Tradition ist, erhellt aus dem Schluss seines Vorworts, in dem er seiner Hoffnung Ausdruck gibt, bei seinen Darlegungen den Pfad kirchlicher Lehre und christlichen Glaubens nicht verlassen zu haben. ${ }^{32}$

Wenn aber die Choralmelodien bereits die Gesetzmäßigkeiten der zwölf Modi erkennen lassen, dann - so der nächste zwingende Schritt für Glarean - setzt dies Verfasser voraus, die eben diesen Regeln bereits beim Verfertigen der Melodien folgten. Solche Komponisten vereinen christliche Tugend und antike Gelehrsamkeit auf vollendete Art und Weise in sich, und Glarean stellt zwei bewunderungswürdige Protagonisten heraus: Notker Balbulus und Hermann den Lahmen. Notkers Pfingstsequenz »Sancti Spiritus assit nobis gratia « lobt er wegen ihrer Würde im Ausdruck, des Variantenreichtums der melodischen Erfindung, die dennoch die Grenzen des Modus nicht sprengt, und wegen der gekonnten Verbindung von Text und Melodie; Erasmus von Rotterdam selbst soll seiner Bewunderung hierfür Ausdruck gegeben haben. ${ }^{33}$ Über Hermann den Lahmen von der Reichenau weiß Glarean

$31 \mathrm{Vgl}$. Sarah Fuller, "Defending the sDodecachordon«: Ideological Currents in Glarean's Modal Theory«, in: Journal of the American Musicological Society 49 (1996), S. 203-205.

32 Dod., Bg. a3 $3^{v}$ : $Q$ Quod ad me attinet, ita hocce negocium me tractasse confido, ut sentiant omnes aequi animi iudices, Christianae pietati ac Ecclesiasticae dignitati ex animo me consulere uoluisse. " ("Was mich betrifft, so vertraue ich darauf, diesen Gegenstand so behandelt zu haben, dass alle unvoreingenommenen Richter einsehen mögen, dass ich um die christliche Frömmigkeit und die Würde der Kirche von ganzem Herzen besorgt war.«)

33 Dod., S. 120f.: "Sed eius exemplum ponamus, prosam, quae in die Pentecostes canitur: Sancti Spiritus adsit nobis gratia. quam impensè placuisse D[omino] ERASMO Roterodamo, et ab eo eximiè olim laudatam memini. ... Habet haec prosa miram modestiam, inenarrabilemque grauitatem, In qua operae pretium est uidere authoris ingenium, quàm uarias in uno Modo inuenerit formulas, quàm limitibus Modi cantum coercitum exhibuerit, quàm eleganter uerba numeris acommodarit« ( $»$ Als Beispiel [für diesen Modus] geben wir die Sequenz, welche an Pfingsten gesungen wird, Sancti Spiritus adsit nobis gratia, die Erasmus von Rotterdam sehr gefallen hat, und, wie ich mich erinnere, von ihm einst sehr gelobt worden ist. ... Diese Sequenz besitzt eine staunenswerte Zurückhaltung sowie eine unbeschreibliche Würde. Es lohnt sich, in diesem Werk den Einfallsreichtum des Autors zu betrachten, wie er verschiedene Formeln in einem einzelnen Modus findet, wie er einen Ge- 
durch Vermittlung eines Angehörigen des Deutschritterordens aus Altshausen erstaunlich viel zu berichten, auch wenn er ihn in das Kloster St. Gallen versetzt und seine Lebensdaten nur schätzen kann. Besonders wichtig ist es ihm, zu betonen, dass Hermann in der Theologie, den quadrivialen Fächern, der Dichtkunst und in den Sprachen gleichermaßen hochbegabt war sowie bemerkenswerte Kompositionen hinterlassen habe. ${ }^{34}$ Vermutlich ohne den Nachdruck zu kennen, mit dem Hermann selbst in seinem Traktat Musica die Verbindung von theoretischer Spekulation und praktischer Musikausübung vertritt, ${ }^{35}$ zeichnet Glarean den Mönch von der Reichenau als Idealtyp der Verschmelzung von hoher Gelehrsamkeit (»eruditio«) mit christlicher Gesinnung ("pietas«). ${ }^{36}$ Wie sehr er Hermann bewundert und wie sehr er sich ihm verbunden fühlt, erhellt auch daraus, dass Glarean anlässlich der Dedikation eines Exemplars seines Dodekachordons an den St. Galler Abt Diethelm Blarer von Wartensee diesen bittet, er möge sein Opus neben die Schriften des St. Galler (sic!) Mönchs Hermannus Contractus in der Klosterbibliothek stellen. ${ }^{37}$ Die marianische Antiphon $» S a l v e$ Regina und die Sequenz »Ave praeclara maris stella« schreibt Glarean der Tradition folgend Hermann zu und bewundert deren unendliche Süße in der melodischen Erfindung. ${ }^{38}$ Besonders bemerkenswert ist, dass hier, wie auch bei der Charak-

sang gestaltet hat, der innerhalb der Grenzen des Modus verbleibt, und wie elegant er die Worte der Melodie anpasst «).

34 Dod., S. 158: "Exempli, quod subnectemus, authorem, Hermannum Contractum ferunt, Comitem à Veringen, Qui fuit monachus Coenobij S. Galli in Heluetijs, quod plurimis Eximijs uiris multis nunc seculis claruit, Hunc addunt in diuinis literis eruditissimum fuisse, nec minus in profanis disciplinis, ut in mathematicis, Oratorijs, \& Poeticis, Linguarum denique peritissimum, Et de Musica ac Monochordo quaedam non indigna lectu scripsisse « ("Als Autor des Beispiels, das wir beifügen werden, gilt Hermannus Contractus, Graf von Veringen, der Mönch des Klosters St. Gallen in der Schweiz war, das nun seit vielen Jahrhunderten durch seine vielen berühmten Männer glänzt. Es wird auch berichtet, dass er in der Theologie äußerst gelehrt gewesen sein soll, und nicht weniger in den weltlichen Wissenschaften, wie Mathematik, Rhetorik und Dichtkunst, und endlich auch besonders erfahren in den Sprachen. Und er soll auch über die Musica und das Monochord manch Lesenswertes geschrieben haben «).

35 M. Richert Pfau, S. J. Morent, Hildegard von Bingen (wie Anm. 7), S. 293.

37 Zum Einfluss des Konzepts der »docta pietas nach Erasmus vgl. Stefano Mengozzi, Between rational theory and historical change in Glareanus's Dodecachordon, $\mathrm{PhD}$ diss. University of Chicago 1998, S. 170 mit Anm. 47.

37 Therese Bruggisser-Lanker, Musik und Liturgie im Kloster St. Gallen in Spätmittelalter und Renaissance, Göttingen 2004 (Abhandlungen zur Musikgeschichte, 13), S. 101 mit Anm. 14.

38 Dod., S. 158: „Certe haec harmonia, cuiuscunque sit, eiusmodi est, ut nihil lepidius fingi, nihil suauius dulciúsue excogitari potuerit.« (»Sicherlich ist diese Harmonia, von wem sie auch stammen mag, dergestalt, dass nichts schöner ersonnen, nichts angenehmer und süßer ausgedacht werden konnte.«). Dod., S. 176: »... Hermannus Comes à Veringen, quem 
terisierung Notkers, vom »ingenium« des Komponisten die Rede ist, einer Auszeichnung, die Glarean sonst nur im Zusammenhang mit den von ihm verehrten Schöpfern mehrstimmiger Musik wie Josquin Desprez, Johannes Ockeghem und Heinrich Isaac verwendet.

Glarean betrachtet Notker und Hermann den Lahmen als Vertreter von Neukompositionen aber nicht etwa isoliert, sondern stellt sie in einen Traditionszusammenhang mit dem älteren Choralrepertoire. Und so erscheinen Ambrosius und Gregor (wie auch Augustinus) als Verfasser der Choralmelodien gleichberechtigt mit den späteren Komponisten von Sequenzen und Offizien. ${ }^{39}$ Für Glarean führt also eine verbindende Linie von den Anfängen zur Spätphase des Choralschaffens, die durchgängig von aus antikem Wissen gespeister Gelehrsamkeit und musikalischer Meisterschaft geprägt ist. In einem solchen Argumentationszusammenhang kann die ältere Vorstellung von einer vagen, inspirierten Herkunft des Chorals keinen Platz mehr haben. Gregor und die heiligen Väter sind nicht mehr nur legendäre Sprachrohre des Heiligen Geistes, sondern Komponisten wie Notker oder Hermann durchaus vergleichbar, und sie alle stehen wiederum in ungebrochener Kontinuität mit dem Altertum. Aus dieser Sichtweise heraus erklärt sich, warum

Contractum nominabant. Homo magni ingenij, ut alibi testati sumus de Ionici Hypoionicique Modorum connexione. Qui mihi in ea prosa de Coelorum regina, IESV CHRISTI Matre, quae Aue praeclara inscribitur, plus musici ingenij ostendisse uidetur, quàm ingens aliorum grex sexcentis cantionum plaustris.« (»[ $\mathrm{Zu}$ diesen ausgezeichneten Männern im Kloster St. Gallen in der Schweiz gehörten der Abt Notker und] Hermannus, Graf zu Veringen, mit dem Beinamen sder Lahmex, ein Mann von großem Geist, wie wir an anderer Stelle bei der Besprechung der Verbindung des ionischen mit dem hypoionischen Modus gezeigt haben. Dieser scheint mir in der Sequenz auf die Himmelskönigin, der Mutter Jesu Christi, die Ave praeclara genannt wird, mehr musikalisches Genie gezeigt zu haben, als die ungeheuer große Schar der anderen in unzähligen Wagenladungen von Gesängen.«)

39 Dod., S. 176: "Ego sanè ad Christianam pietatem, qua prisci Ecclesiastici uiguerunt, plurimum simplicem cantum, per Modos eruditè distinctum, conferre arbitror, nec parum facere ad animorum (ut nunc loquimur) deuotionem: maxime qualeis apud Italos Ambrosius instituit. Gregorius item, ac Augustinus, Ecclesiae lumina. Deinde apud Gallos ac Germanos eximij uiri, è quorum numero fuêre in Coenobio S[ancti] Galli in Heluetiis Nockerus Abbas, \& Hermannus Comes à Veringen, quem Contractum nominabant." (»Ich glaube fest, dass der einfache Gesang, der geschickt durch die Modi unterschieden wird, sehr viel zur christlichen Frömmigkeit beitragen kann, in welcher die frühen kirchlichen Musiker stark waren; auch trägt er nicht wenig zur geistigen Andacht (wie man jetzt sagt) bei, ganz besonders solche Gesänge, wie sie Ambrosius bei den Italienern eingeführt hat, und ebenso Gregor und Augustinus, die Leuchten der Kirche. Dann auch bei den Franzosen und Deutschen ausgezeichnete Männer, aus deren Reihen im Kloster St. Gallen in der Schweiz der Abt Notker und Hermannus, Graf zu Veringen, mit dem Beinamen sder Lahme«, stammten.«) 
Glarean in dem eingangs zitierten Passus von den Messgesängen und vom Choral im Allgemeinen als dem Höhepunkt menschlichen Schaffens spricht. Der Umstand, dass Glarean die Gesetzmäßigkeiten seines Modalsystems in den überlieferten Choralmelodien aufweisen kann, wird in seiner Vorstellung nur dadurch möglich, dass diese von Anfang an durch verständige Komponisten so verfasst wurden.

Damit erhält der Choral an sich aber auch eine wesentliche Aufwertung, und hierin ist das zweite wichtige Anliegen Glareans zu sehen. Im Zeichen ausgeprägter choralfeindlicher Anwürfe, die sowohl von reformatorischen Kreisen als auch von Verfechtern des Primats mehrstimmiger Kirchenmusik ausgehen, sieht sich Glarean zu einer Parteinahme für den Choral herausgefordert. Sebald Heyden, von dem Glarean zahlreiche Musikbeispiele in sein Dodekachordon übernahm, hält beispielsweise den Choral eher für überflüssig, wenn nicht für schädlich; auch sieht er in der Chorallehre wenig Nutzen für die Figuralmusik. ${ }^{40}$ Umgekehrt verfasst 1561 der St. Galler Konventuale Mauritius Enck auf Geheiß seines Abts für die neu für das Kloster angelegten Chorbücher mit mehrstimmigen Vertonungen von Manfredus Barbarini Lupus eine »Praefatio«, in der er unter Bezugnahme auf Glareans Dodekachordon dem einstimmigen Choral eine zumindest gleichrangige Stellung gegenüber der mehrstimmigen Figuralmusik zuerkennt. ${ }^{41}$ Dass Enck, der wohl bei Glarean studierte, ${ }^{42}$ hier keinesfalls eine allgemeine Einschätzung wiedergibt, zeigt z. B. die 1553 bzw. 1563 und 1568 in Straßburg unter dem Titel Bellum musicale inter plani et mensurabili cantus reges de principatu in Musicae provincia obtinendo contendentes erschienene Streitschrift des Metzer Organisten Claudius Sebastiani. ${ }^{43}$

Glarean nimmt in diesen Streitfragen eine vermittelnde Rolle ein: Einund mehrstimmige Musik besitzen ihre je eigene Wichtigkeit und Würde, und ihre jeweiligen Schöpfer, die "phonasci« und "symphonetae", sollten nicht gegeneinander ausgespielt werden ${ }^{44}$ Vielmehr sind die seit ihren An-

40 Vgl. Sebald Heyden, De arte canendi, Nürnberg 1540, Reprint New York 1969 (Monuments of Music and Music Literature in Facsimile, 2.139), S. 4 und 136. Vgl. auch Alfred Kosel, Sebald Heyden (1499-1561). Ein Beitrag zur Geschichte der Nürnberger Schulmusik in der Reformationszeit, Würzburg 1940 (Literarhistorisch-musikwissenschaftliche Abhandlungen, 7), S. 28; und Clement A. Miller, »Sebald Heyden's $>$ De arte canendi<: Background and contents", in: Musica Disciplina 24 (1970), S. 81f. und 96.

41 Th. Bruggisser-Lanker, Musik und Liturgie (wie Anm. 37), S. 98-101.

42 Ebda., S. 93.

43 Vgl. ebda., S. 108-113.

44 Dod., S. 178: „Ob has igitur, ac antea dictas causas ego eximios Phonascos neutiquam Symphonetis postposuero: sed neque Ecclesiasticum cantum, arte uera, ac Modis naturali- 
fängen aus der glücklichen Verbindung von "eruditio « und "pietas « hervorgegangenen Choralmelodien die Voraussetzung dafür, dass sie die hervorragenden Meister polyphoner Musik zur Grundlage ihrer Werke erwählt haben.

Ob Glareans Sichtweise auf den Choral eine smoderne $\mathrm{zu}$ nennen wäre? Jedenfalls weist er mit seiner Verknüpfung des älteren Choralrepertoires mit den späteren Neuschöpfungen im Zeichen des Kompositionsbegriffs in verblüffender Weise auf eine Erkenntnis erst der jüngeren Choralforschung voraus: dass nämlich auch der Choral nicht aus der Kompositionsgeschichte herausgenommen werden kann. ${ }^{45}$

bus constantem cedere puto oportere multarum uocum garritui. Vtrumque in honore atque sua, qua apud ueteres uiguêre, \& hodie sunt, authoritate ac existimatione permanere uelim.» ("Deshalb, aus diesen und den zuvor erwähnten Gründen, schätze ich ausgezeichnete Verfasser einstimmiger Melodien keinesfalls geringer als Komponisten mehrstimmiger Musik. Ebenso glaube ich, dass der kirchliche Gesang, der auf wahrer Kunst und naturgemäßen Modi beruht, nicht dem vielstimmigen Gezwitscher weichen müsse. Ich wünsche, dass beide in der Ehre, in dem Ansehen und in der Achtung verbleiben, die sie bei den Alten genossen, und in der sie sich heute noch befinden. «)

45 Vgl. K. Schlager, Ars cantandi (wie Anm. 14), S. 220; und Andreas Traub, "Zur Kompositionslehre im Mittelalter «, in: Beitrüge zur Gregorianik 17 (1994), S. 63. 